\title{
El neuromarketing aplicado a los tráileres cinematográficos del año 2009 al 2013*
}

\section{The neuromarketing applied to movie trailers of the years 2009 to 2013}

\author{
Francisco de Paula Contreras Albornoz ${ }^{* *}$ \\ Luis Fernando Gasca Bazurto***
}

\begin{abstract}
Resumen
En el presente artículo se realiza un diagnóstico global sobre la realidad del neuromarketing aplicado a los tráileres cinematográficos del año 2009 al 2013. El método de la investigación fue documental y la modalidad: estado del arte. La investigación se fundamentó sobre la revisión de textos escritos, principalmente libros, revistas especializadas, artículos, congresos, etc. Sin embargo, se incluyeron algunas publicaciones no científicas, ya que eran deinterés para esta investigación. El objetivo fue establecer un cuerpo que permitiera precisar el fenómeno, comprender la relación con la cinematografía y en particular con los tráileres cinematográficos. La principal conclusión del estado del arte propuesto en el periodo estudiado, es que es una metodología que acoge las neurociencias y el marketing. Respecto a la cinematografía, el neuromarketing se desarrolló en Hollywood con el objetivo de comprender el gusto de los espectadores para producir tráileres más atractivos.
\end{abstract}

Palabras clave: Cerebro, cine, neurocinema, neuromarketing, tráiler.

El presente artículo es producto de investigación, cuyo objetivo fue elaborar el estado del arte del neuromarketing aplicado a los tráileres cinematográficos entre los años 2013 a 2009. Como primera etapa de una investigación más amplia que busca indagar los métodos del neuromarketing para la producción de tráileres cinematográficos.

** $\quad$ Profesional en publicidad. Universidad Jorge Tadeo Lozano. Candidato a Máster en Escrituras Creativas de la Universidad Nacional de Colombia. Profesor investigador. Corporación Unificada Nacional - CUN, grupo CODIM. franzsor@gmail.com, tel.: 57315 5062333 .

**** Realizador de Cine y televisión. Universidad Nacional de Colombia. Candidato a Máster en Literatura y Cultura del Instituto Caro y Cuervo. Profesor investigador. Corporación Unificada Nacional - CUN. Grupo CODIM. luisf.gascab@gmail.com, tel.: 573013053966. 


\begin{abstract}
State of the art: In this article a global assessment of the reality of neuromarketing applied to movie trailers from 2009 to 2013. The research method was documentary mode is performed. The research was based on the review of written texts mainly books, journals, articles, conferences, etc. However, somenon-scientific publications were included as they were relevant to this investigation. The aim was to establish a body that would define the phenomenon, understand the relationship with film and particularly with the movie trailer. The main conclusion of state proposed in the period studied is that art is a methodology that welcomes neuroscience and marketing. Regarding the film, the Hollywood neuromarketing developed in order to understand the taste of the spectators to produce more attractive trailers.
\end{abstract}

Keywords: Brain, movies, neurocinema, neuromarketing, tráiler.

\title{
1. Introducción
}

Los descubrimientos en el campo de las neurociencias y la diversificación de estas en diversas áreas del conocimiento como la economía y la publicidad, sumado a los avances tecnológicos, ha conducido al surgimiento del denominado neuromarketing. Dicha denominación corresponde a metodologías que buscan predecir el comportamiento de los consumidores con base en técnicas de las neurociencias. Las técnicas de las neurociencias que se aplican a la producción cinematográfica se designan neurocinema.

El neuromarketing se ha publicitado bastante y ha despertado el interés a diferentes niveles. Sin embargo, el exceso de divulgación comercial y el auge de los gurúes que venden libros, seminarios y conferencias, llevó a los investigadores a interesarse en el tema e indagar si es un mito, un fenómeno, una moda o incluso un nuevo campo de conocimiento. Los hallazgos de la investigación podrían contribuir a abrir una línea de investigación en el terreno de los medios audiovisuales.

En el curso del primer semestre del 2014 se llevó a cabo la búsqueda y revisión documental, con el objetivo de comprender 
el alcance del concepto neuromarketing y la influencia de dicha práctica en la cinematografía y, en particular, en la producción de los tráileres cinematográficos. A continuación se clasificó, catalogó y analizó el material con base en el cual se elaboró un documento que se denominó Estado del arte del neuromarketing aplicado a los tráileres cinematográficos entre los años 2009 al 2013. El período de tiempo que se fijó para realizar la búsqueda incluyó el estreno de la película Avatar (Cameron, 2010), una de los primeros filmes en el que se aplicaron técnicas de las neurociencias con un fin comercial. De acuerdo con Randall (2011) el director de cine James Cameron, director de la película Avatar (Cameron, 2010), se asoció con la compañía de neuromarketing MindSing para efectuar el escáner cerebral a un grupo de individuos, con el objetivo de analizar el nivel de aceptación del movie tráiler de la película.

El neuromarketing aplicado a los tráileres cinematográficos es un área muy específica y fue difícil hallar información detallada y especializada. La investigación también incluye diversas fuentes de carácter divulgativo no científico, ya que contribuían a comprender el panorama o conducían a documentos más precisos. Los resultados alcanzados no son definitivos; sin embargo, el documento logra presentar el origen del neuromarketing, definir el concepto, proponer un panorama, relacionar el neuromarketing con la cinematografía y comprender los motivos por los cuales el neuromarketing se aplica a la producción de los tráileres cinematográficos.

\section{Metodología}

El método fue revisión documental y la modalidad estado del arte. La investigación se desarrolló en cuatro fases: búsqueda, clasificación, interpretación de la información y redacción del documento. Los resultados obtenidos se estructuraron de acuerdo con la relevancia de las fuentes. Se fijaron límites y criterios de búsqueda de información. Se dio prioridad a las fuentes primarias que se recopilaron en libros 
y artículos científicos. Sin embargo, se determinó que de no hallarse información en relación con los criterios de búsqueda se procedería a consultar fuentes secundarias.

\subsection{Límites de búsqueda}

\subsubsection{La búsqueda se delimitó a las fechas del 2009 al 2013.}

\subsubsection{Palabras clave.}

Se escogieron las palabras neuroscience, para neurociencia; neuromarketing, para la unión entre marketing y neurociencia; neurocinema, para la unión entre neurociencia y cine; cinematography, para lo relacionado con el arte cinematográfico; emotions tráiler, para la relación entre los avances de las películas y las emociones que implican; movie tráiler, para los avances de las películas que se exhiben en los medios de comunicación audiovisual.

\subsection{Fuentes de información.}

Se emplearon los recursos electrónicos disponibles en la biblioteca central Jaime Alberto Rincón Prado de la Corporación Unificada Nacional de Educación Superior (CUN) y, de manera abierta, se indagó en la web. En primer lugar, en la biblioteca Jaime Alberto Rincón Prado se consultaron las bases de datos: Ebscohost, Business Searching Interface, ProQuest. Externo a la universidad se consultó la base Jstor y Google Académico. En segundo lugar, se consultó en los repositorios web en línea de la Universidad del Rosario y Universidad Nueva granada, ya que no ofrecían restricciones de consulta. En tercer lugar, se revisó de manera abierta la web en búsqueda de revistas y periódicos de publicidad, economía, negocios y cinematografía. En cuarto lugar, se consultó en sitios web y blogs relacionados con los temas mencionados en el punto anterior. Los documentos consultados incluyeron algunas tesis de grado de estudiantes de publicidad y negocios. 


\subsection{Instrumentos.}

\subsubsection{Google drive.}

El principal instrumento que se empleó fue Google drive que permitía la colaboración en línea. A continuación se creó una carpeta para guardar documentos y se creó una hoja de cálculo. La carpeta se creó con el objetivo de guardar los documentos que se pudieran descargar de la web. Dentro de la carpeta principal se crearon carpetas que se nombraron de acuerdo a las palabras clave y, en estas últimas, se crearon nuevas carpetas para almacenar la información por cada año de consulta, a saber: 2009, 2010, 2011, 2012 y 2013.

La hoja de cálculo se dividió en columnas, en las que se ubicó la información de acuerdo con los criterios: Fecha en que se ubicó el documento, título del documento, título de la publicación, fecha de publicación, volumen, número, ISSN, ISBN, autor o autores, idioma original del documento, resumen del texto, páginas (páginas en las que se encontraran conceptos relacionados con las palabras clave), relevancia (conceptos relacionados con las palabras clave). A continuación, las columnas designaban las categorías del documento en: libro, artículo científico, tesis o disertación, artículo de prensa, texto en web, texto en blog, documento en línea, documento impreso. Para terminar, en la última columna se transcribía la dirección online o física del documento.

\subsection{Criterios de selección}

Se elaboró una lista de objetivos para establecer los niveles de relevancia.

1. Clasificar los documentos en fuentes científicas y fuentes de divulgación no científica.

2. Clasificar las fuentes del ítem (1) en primarias y secundarias.

3. Clasificar las fuentes primarias y secundarias de acuerdo con el orden propuesto para las palabras clave. 
A continuación se clasificaron los documentos y se elaboraron resúmenes analíticos para proceder a la fase de interpretación de la información y redacción del documento.

\section{Resultados}

\subsection{Concepto y ramas de las neurociencias}

Según lo expuesto por Medical New Today, MNT (2013), las neurociencias son el conjunto de estudios del cerebro para comprender la influencia que este ejerce sobre las funciones cognitivas y del comportamiento humano, con el fin de encontrar soluciones a los trastornos neurológicos, psiquiátricos y del desarrollo neurológico. De acuerdo con la Society of neuroscience (2015), los neurocientíficos son un grupo multidisciplinario de profesionales que intentan descifrar y comprender la manera en que funciona el cerebro y el sistema nervioso del ser humano. "La revolución de las neurociencias inició cuando estos científicos se dieron cuenta que para discernir sobre el funcionamiento del cerebro se necesitaba un posicionamiento multidisciplinar, requiriendo conocimientos sobre multiplicidad de hechos". (Soriano \& Guillazo, 2007, p. 13).

De acuerdo con MNT (2013), desde la década de 1950 el estudio científico del cerebro y del sistema nervioso - de forma paralela los avances en el campo de la neurociencia computacional, electrofisiología y biología molecular- han estado en progreso continuo, por lo tanto los neurocientíficos han logrado profundizar en el estudio de la estructura del sistema nervioso, sus funciones, anomalías u otras alteraciones. Lo expuesto ha condicionado a que el estudio del cerebro se despliegue en diversidad de especialidades. La tabla 1 presenta las principales ramas de las neurociencias de acuerdo con MNT (2013). 
Tabla 1. Ramas de las neurociencias.

\begin{tabular}{|c|c|}
\hline Neurociencia afectiva & Estudio de las neuronas en relación con las emociones. \\
\hline $\begin{array}{l}\text { Neurociencia } \\
\text { conductual }\end{array}$ & Estudio de cómo el cerebro afecta el comportamiento. \\
\hline Neurociencia celular & $\begin{array}{l}\text { Estudio de la forma y propiedades de las neuronas a } \\
\text { nivel celular. }\end{array}$ \\
\hline Neurociencia clínica & Estudio de los trastornos del sistema nervioso. \\
\hline $\begin{array}{l}\text { La neurociencia } \\
\text { cognitiva }\end{array}$ & $\begin{array}{l}\text { Estudio de las funciones cognitivas superiores en los } \\
\text { seres humanos. }\end{array}$ \\
\hline $\begin{array}{l}\text { Neurociencia } \\
\text { computacional }\end{array}$ & $\begin{array}{l}\text { Estudio de la aplicación de las matemáticas, la física } \\
\text { y otros campos computacionales para aprender el } \\
\text { funcionamiento del cerebro }\end{array}$ \\
\hline Neurociencia Cultural & $\begin{array}{l}\text { Estudia cómo las creencias, prácticas y valores } \\
\text { culturales son moldeadas por el cerebro, la mente y } \\
\text { los genes. }\end{array}$ \\
\hline $\begin{array}{l}\text { La neurociencia del } \\
\text { desarrollo }\end{array}$ & $\begin{array}{l}\text { Estudia el sistema nervioso sobre la base celular, para } \\
\text { comprender cuáles son los mecanismos subyacentes } \\
\text { que existen en el desarrollo neuronal. }\end{array}$ \\
\hline Neurociencia molecular & $\begin{array}{l}\text { Estudio de la función de las moléculas individuales en } \\
\text { el sistema nervioso. }\end{array}$ \\
\hline Neuroingeniería & $\begin{array}{l}\text { Estudia las técnicas de ingeniería para reemplazar, } \\
\text { reparar o mejorar los sistemas neuronales. }\end{array}$ \\
\hline Neuroimagen & $\begin{array}{l}\text { Busca el desarrollo técnico para ver la imagen del } \\
\text { cerebro a partir de dispositivos tecnológicos para } \\
\text { examinar a los pacientes sin necesidad de invadir el } \\
\text { cuerpo del individuo. }\end{array}$ \\
\hline Neuroinformática & $\begin{array}{l}\text { Estudia la integración de datos en las áreas de } \\
\text { la neurociencia para entender el cerebro y tratar } \\
\text { enfermedades. }\end{array}$ \\
\hline Neurolingüística & $\begin{array}{l}\text { Estudia los mecanismos neuronales del cerebro que } \\
\text { controlan la adquisición, comprensión y expresión del } \\
\text { lenguaje. }\end{array}$ \\
\hline Neurofisiología & $\begin{array}{l}\text { Estudia la relación del cerebro con sus funciones y la } \\
\text { relación con el resto del organismo. }\end{array}$ \\
\hline Paleoneurology & Estudio del cerebro con base en fósiles. \\
\hline La neurociencia social & $\begin{array}{l}\text { Estudio interdisciplinario dedicado a la comprensión } \\
\text { de cómo los sistemas biológicos implementan procesos } \\
\text { sociales y de comportamiento. }\end{array}$ \\
\hline $\begin{array}{l}\text { Sistemas de } \\
\text { neurociencia }\end{array}$ & $\begin{array}{l}\text { Estudia las vías de flujo de datos dentro del sistema } \\
\text { nervioso central para definir cómo se produce allí el } \\
\text { proceso. }\end{array}$ \\
\hline
\end{tabular}

Fuente: MNT (2013). 


\subsection{Concepto y técnicas del neuromarketing}

El neuromarketing es la integración de los conceptos, métodos y dispositivos de las neurociencias con los conceptos y métodos y recursos del marketing. En particular el marketing: "Para decirlo con sencillez el marketing es gestionar relaciones redituables con los clientes. La meta del marketing es crear valor para los clientes y captar, en reciprocidad, el valor de los clientes" (Kotler \& Armtrong, 2013, p. 2). Para Tirado (2013),

\footnotetext{
El marketing es una actividad fundamental en las empresas. Su estudio se centra en el intercambio, por lo que su área de interés engloba el análisis de las relaciones de una empresa con los diferentes agentes del entorno que interactúan con ella (p. 9).
}

Para la American Marketing Association, "Marketing is the activity, set of institutions, and processes for creating, communicating, delivering, and exchanging offerings that have value for customers, clients, partners, and society at large" (AMA, 2013, párr.1). Es decir, el marketing es la actividad y el conjunto de decisiones y procesos para crear, comunicar, entregar e intercambiar bienes de valor con los clientes, los socios y la sociedad en general.

El neuromarketing es una metodología que emplea técnicas de las neurociencias para la investigación de mercados, según coinciden Braidot (2010), Lindstrom (2010) Smidts (2002), Malfitano (2007), Pradeep (2010) y Lehrer (2011). Para Berčík, Horská, Wang y Chen, (2015) el neuromarketing es un enfoque interdisciplinar que emplea técnicas de las neurociencias para comprender las emociones humanas con base en las cuales se crean estrategias de marketing eficientes. Los investigadores en neuromarketing buscan medir la actividad del cerebro para analizar las zonas que se activan para comprender los sistemas neuronales que intervienen con base en los cuales idean estrategias para vender productos de manera más eficiente.

"Los últimos avances en las neurociencias, especialmente, las investigaciones de los doctores Antonio Damasio y Joseph LeDoux 
han demostrado que la toma de decisiones no es un proceso racional" (Braidot, 2010, p. 9). De acuerdo con Damasio (1994), un evento externo detona mecanismos cognitivos que, en conjunto, crean una reacción corporal. Un evento puede ser el encuentro con una serpiente o reconocer en una fotografía a un ser querido. Los especialistas en mercadeo utilizan las técnicas de las neurociencias para descubrir qué incidentes conducen a una persona a decidir entre una marca $\mathrm{u}$ otra. De acuerdo con Linstrom (2010) “(...) cuando vemos y olemos simultáneamente hay algo que nos agrada -como el talco Johnson para niños combinado con el aroma clásico de vainilla-, varias regiones del cerebro se activan simultáneamente” (p. 161). En consecuencia, los expertos en neuromarketing emplean técnicas para registrar las respuestas neurofisiológicas de los individuos a estímulos externos para comprender los deseos de compra.

\subsubsection{Técnicas de neuromarketing.}

Los especialistas en neuromarketing emplean diversas técnicas para analizar las reacciones emocionales de los sujetos. La mayoría de las técnicas emplean dispositivos que registran y miden la actividad cerebral. Las técnicas de las neurociencias que se emplean en marketing se dividen en dos grupos. En primer lugar, están las técnicas que registran y miden la actividad cerebral. En segundo lugar, están las técnicas que registran las acciones y reacciones del organismo. Dichas categorías se componen de los siguientes métodos.

3.2.1.1. Técnicas de registro de la actividad cerebral

Técnicas que emplean dispositivos que registran la actividad directamente del cerebro a diferentes niveles de profundidad.

3.2.1.1.1. EEG, Electroencefalografía

En el área de neurometría la electroencefalografía o EEG ha sido clave para el desarrollo de las neurociencias y, en particular, del neuromarketing, según lo expuesto por Vecchiato (2013), Morin (2011) 
y Madan (2010). De acuerdo con Palacios (2002) en 1770 el italiano Luigi Galvani publicó las primeras observaciones sobre la electricidad animal. El 6 de julio de 1924, Hans Berger -Jefe de la Unidad de Psiquiatría de la Universidad de Jena (Alemania) - realizó el primer registro de oscilaciones rítmicas del cerebro a una joven de 16 años de nombre Zedel. El doctor Berger se considera el padre de la EEG. Un equipo de electroencefalografía captura el registro de la actividad de los lóbulos cerebrales a nivel subcortical a través de electrodos que se fijan sobre el cuero cabelludo del individuo.

3.2.1.1.2. fMRI, Resonancia magnética funcional de imágenes

De acuerdo con Hasson, Landesman, Knappmeyer, Vallines, Rubin y Heeger (2008), el fMRI es un dispositivo que mide la actividad cerebral de las regiones más profundas del cerebro, por ejemplo el núcleo acumbens. El aparato es un gran cilindro en el que se introduce e inmoviliza a un individuo al que se le coloca un casco que registra la actividad neuronal. El dispositivo registra los potenciales evocados del cerebro y los interpreta en imágenes que representan la citoarquitectura cerebral.

\subsection{MEG, Magneto encefalografía}

De acuerdo con Benito y Guerra (2011), el MEG capta los campos magnéticos de la actividad coordinada de las neuronas y registra la más pequeña actividad. Este dispositivo se usa principalmente para investigar las relaciones entre las estructuras cerebrales y sus funciones.

\subsection{PET, Tomografía de Emisión de Positrones}

Es una técnica invasiva que utiliza una sustancia radiactiva llamada marcador radioisótopo que se introduce en el cuerpo del sujeto a través de una inyección. Un escáner PET detecta la radiación gamma que genera el marcador y reproduce una imagen del metabolismo de la glucosa en el cerebro para detectar las áreas de mayor actividad cerebral, de acuerdo a lo expuesto por Berrocal (2013) y García (2014). 
3.2.1.2. Técnicas que registran las acciones y reacciones del organismo.

Son técnicas que emplean dispositivos que registran indicadores corporales que permiten deducir la respuesta emocional de los sujetos sin medir directamente la actividad cerebral.

\subsection{EMG, Electromiografía}

De acuerdo con Benito y Guerra (2011) el EMG es un dispositivo que consta de un conjunto de pequeños electrodos de bajo voltaje que se fijan al rostro de un sujeto. Los electros se ubican sobre la epidermis cerca a los músculos y terminales nerviosas. El EMG registra la actividad eléctrica de los músculos esqueléticos ${ }^{1}$ para calcular la respuesta emocional.

\subsection{Eye tracking}

De acuerdo con Benito y Guerra (2011), el Eye tracking registra los puntos de atención sobre los que se fija la mirada de un sujeto. Los ojos crean recorridos visuales que permiten crear mapas. Una persona observa superficies pero los ojos se detienen un instante en los lugares que le interesaron, estas áreas se denominan "puntos calientes”. Los "puntos calientes" permiten inferir si existe atracción hacia lo que se está observando. Los dispositivos de Eye tracking capturan el movimiento de los globos oculares, la dilatación de la pupila y el parpadeo del sujeto en relación con el movimiento de la cabeza.

\subsection{Respuesta galvánica}

La técnica GSR o SRC es una tecnología que se basa en el polígrafo o detector de mentiras. De acuerdo con Ramanchandram y Hirstein (1999), un equipo de registro de respuesta galvánica capta el nivel de excitación de la piel de un sujeto que tiene estímulos sensoriales. En la misma categoría se encuentran los equipos que captan la activación

1 Músculos esqueléticos, musculaquis o estriados, son aquellos músculos que están unidos al esqueleto. 
electrodermal (EDA) o activación electrodermal que mide el nivel de calor de la epidermis. Los cambios en la piel se producen por el estímulo de glándulas sudoríparas que reaccionan por un estímulo emocional.

La tabla 2 presenta las técnicas, ya mencionadas, que se emplean en el neuromarketing.

Tabla 2. Técnicas utilizadas en el neuromarketing.

\begin{tabular}{|l|l|}
\hline Técnicas de neuro - métrica & Técnicas alternativas \\
\hline EEG, Electroencefalografía & EMG, Electromiografía \\
\hline fMRI, Resonancia magnética funcional de imágenes & Eye tracking \\
\hline MEG, Magneto encefalografía & GSR o SRC, Respuesta galvánica \\
\hline PET, Tomografía de Emisión de Positrones & \\
\hline
\end{tabular}

Las técnicas de neuromarketing se popularizaron porque permitían comprender, desde la ciencia, la manera en que funciona el cerebro de los consumidores. Asimismo, la mayoría de las nuevas tecnologías facilitan el registro de la actividad neuronal de los sujetos de forma no invasiva. La mayoría de dispositivos registran la actividad y comunicación entre las células nerviosas que se produce por electroquímica. De acuerdo con Maestú, Quesney, Ortiz, Fernández, Campo y Amo (2003), los estímulos del mundo exterior se perciben con los cinco sentidos y generan progresiones de señales eléctricas, magnéticas y químicas que alcanzan a registrarse con técnicas EEG o electroencefalografía. Existen diversidad de prácticas desarrolladas por los neurocientíficos, y los investigadores en neuromarketing las han utilizado.

\subsection{Concepto del tráiler cinematográfico.}

El término tráiler es un anglicismo problemático en particular para indagar sobre el tema propuesto, pues el término se confunde con los tráiler que arrastran los camiones. En el escenario hispanohablante también se habla de avance o promocional. De acuerdo con Dornaleteche (2007), "Andrew J. Kuehn, posiblemente la persona más influyente en la historia del tráiler cinematográfico define el tráiler como una 
herramienta de marketing que posiciona un producto -en este caso, una película- en el mercado -en este caso, el cinematográfico" (p. 100). Según Kernan (2004), los tráiler son los avances de la película próxima a estrenarse que se incluían en la misma lata al final de la cinta:

The first trailer was shown in 1912 at Rye Beach, New York, which was an amusement zone like Coney Island. One of the concessions hung up a white sheet and showed the serial "The Adventures of Kathlyn". At the end of the reel Kathlyn was thrown in the lion's den. After this "trailed" a piece of film asking Does she escape the lion's pit? See next week's thrilling chapter! Hence, the word "trailer", an advertisement for an upcoming picture. They've tried calling them Previews or Prevues of Coming Attractions, but everybody in the trade calls them trailers (p. 27).

Pons (2012) coincide con Kernan (2004) y con Lienhart, Pfeiffer y Effelsberg (1997) en que el tráiler es un formato de publicidad que se emplea para promocionar un filme. Se denomina tráiler porque dicha pieza promociona y arrastra al público en el sentido de crearle el deseo de ver la película en el momento que salga al mercado.

\subsection{Aplicación de las neurociencias al tráiler cinematográfico}

Según Gardner (2009), en el año 2004 el Dr. Uri Hasson realizó un trabajo postdoctoral para Neural Science (CNS) de la Universidad de Nueva York. En el año 2008 el Dr. Hasson publicó los hallazgos en el artículo "Neurocinematics: The Neuroscience of Film", en este documento se emplea por primera vez el término neurocinematics. Hasson et al. (2008) realizaron un estudio de resonancia magnética funcional (fMRI) para comprender qué ocurría en el cerebro de los espectadores que observaban un filme. Hansson et al. (2008) utilizaron para el experimento las películas Il buono, il brutto, il cattivo (1966), dos comedias de Chaplin, un tele film de suspenso, un documental y un audiolibro. Hasson et al. (2008) concluyeron que ciertos tipos de películas provocan mayor activación del sistema límbico del espectador. De acuerdo con Haines (2002) se ha reconocido que en la citoarquitectura cerebral el sistema límbico es el área en la cual se procesan las emociones. En la tabla 3 se resume la experiencia del Dr. Hasson. 
Tabla 3. Resumen del estudio de Hansson et al. (2008)

\begin{tabular}{|l|l|c|l|c|}
\hline \multicolumn{1}{|c|}{ Género } & \multicolumn{1}{|c|}{ Nombre } & Año & \multicolumn{1}{c|}{ Autor } & ISC \\
\hline Audio-libro & Alice in Wonderland & 1865 & Lewis Caroll & $10 \%$ \\
\hline Ficción & The Adventurer & 1917 & $\begin{array}{l}\text { Charles } \\
\text { Chaplin }\end{array}$ & $25 \%$ \\
\hline Ficción & City Lights & 1931 & $\begin{array}{l}\text { Charles } \\
\text { Chaplin }\end{array}$ & $30 \%$ \\
\hline Ficción & Alfred Hitchcock Presents: Bang! Your Dead. & 1961 & $\begin{array}{l}\text { Alfred } \\
\text { Hitchcock }\end{array}$ & $67 \%$ \\
\hline Ficción & Il buono, il brutto, il cattivo & 1966 & Sergio Leone & $45 \%$ \\
\hline $\begin{array}{l}\text { Ficción/ } \\
\text { Comedia }\end{array}$ & Curb Your Enthusiasm & 2000 & Larry David & $18 \%$. \\
\hline Documental & One shot Washington Square Park & 2004 & No registra & $12 \%$. \\
\hline
\end{tabular}

De acuerdo con Hasson et al. (2008), el ISC es una media que intenta correlacionar el nivel emocional de un grupo de espectadores que observan un filme. El ISC, desde el punto de vista cualitativo, permite medir y cotejar el registro de la actividad neuronal del cerebro de los espectadores para evaluar si la mayoría concuerda en el nivel de impacto emocional que les produjo el filme. El mayor porcentaje ISC significa que se activan más áreas del cerebro del observador durante la experiencia. El ejercicio demostró que existe proporción respecto al nivel emocional que experimentaron los espectadores (ISC) durante la apreciación de cada pieza audiovisual, al igual que en el audio libro Alice in Wonderland (Caroll, 1865). El tele filme Alfred Hitchcock Presents: Bang! you died (Hitchcock, Swanton \& Vosper, 1961) alcanzó un ISC de $67 \%$, el mayor porcentaje entre los espectadores, en consecuencia esta pieza produjo que se activaran mayor proporción de áreas del cerebro que las demás.

The fact that Hitchcock was able to orchestrate the responses of so many different brain regions, turning them on and off at the sametime across all viewers, may provide neuroscientific evidence for his notoriously famous ability to master and manipulate viewers' minds. Hitchcock often liked to tell interviewers that for him "creation is based on an exact science of audience reactions” (Hasson, et al., 2008, p. 16). 
Hansson, et al. (2008) exponen que se puede considerar a Alfred Hitchcock un gran director de orquesta por su habilidad para inducir respuestas en diferentes regiones del cerebro. Las películas del director en mención podrían proporcionar evidencia neurocientífica para comprender la manera en que lograba manipular emocionalmente a los espectadores, ya que concebía la creación a la manera de una ciencia exacta que le permitía hacer reaccionar al público.

De acuerdo con Randall (2011), el neuromarketing emplea técnicas de las neurociencias para evaluar el registro de la actividad del cerebro de los espectadores para comprender la respuesta emocional que le generan los tráileres cinematográficos, en este sentido para los grandes estudios de Hollywood el neuromarketing es una potente herramienta para predecir la taquilla. Según Randall (2013), Fox utilizó la biometría y la neurometría para la producción de los tráileres de las películas Die Hard 4: Live Free or Die Hard (Wiseman, 2008) y Rango (Verbinski, 2011); esta última generó un ingreso de taquilla de US\$ 245 millones. Randall (2013) expone que Innerscope Research realizó una investigación sobre la efectividad de los tráileres cinematográficos y descubrió que una película cuyo tráiler alcance en el público un umbral emocional del $65 \%$ es probable que genere hasta US\$ 10 millones de ingresos en taquilla el primer fin de semana. De igual manera, un tráiler que logre el umbral del 80\% es muy probable que gane más de US\$ 20 millones el primer fin de semana. De acuerdo con lo dicho, Carl Marci de CEO of Innerscope tells Fast Company le explicó a Randall (2013) que:

The study's real value to the film industry is in putting this new method to work. Biometrics can be used to evaluate trailer creative up to eight weeks prior to movie launch--enough time to rework the trailer creative based on high and low points in the target audience's emotional experience, put the promotion back into market, and improve the ultimate performance on opening weekend. (párr. 3). 
De acuerdo con Randall (2013), Carl Marci de Innerscope expone que el neuromarketing es un nuevo y valioso método de trabajo, ya que las técnicas que se emplean permiten evaluar un tráiler cinematográfico antes del lanzamiento de la película y evaluar si la respuesta del público es positiva antes de que el filme se estrene en pantalla.

\subsection{La relación entre las neurociencias, el movie tráiler y las emo- ciones}

De acuerdo con Hasson et al. (2008), la tecnología EEG es determinante para medir la actividad cerebral de un individuo que observa un filme. El registro EEG de la actividad cerebral de un espectador que observa un tráiler cinematográfico permite distinguir las áreas del cerebro que se activan, en consecuencia se podría determinar cuáles secciones influyen sobre las emociones para calcular la recepción del público objetivo. De acuerdo con Damasio (1994), “(...) sentir tus estados emocionales, que equivale a decir que estás consiente de las emociones te ofrece una respuesta flexible basada en la respuesta particular de tus interacciones con el medio ambiente" (p. 157). Es decir, el entorno expone a los sujetos a diferentes situaciones que pueden provocar emociones. Por ejemplo, el reencuentro con un ser querido produce emoción en las dos personas involucradas, esta es una emoción positiva. Por otro lado, la realidad que un sujeto percibe desagradable genera una emoción negativa. Según Damasio (1994), una emoción activa el sistema límbico que secreta hormonas que se manifiestan a nivel somático, por ejemplo una emoción positiva activa el núcleo accumbens que libera dopamina, dicha hormona actúa en el organismo a la manera de un relajante.

Martin Lindstrom explica a Spurlock (2011) que la efectividad de un tráiler y de una película se mide con base en los registros de respuesta de la actividad neural; si la actividad es alta significa que el espectador esta más implicado emocionalmente. Se espera que una película o un tráiler muestre un comportamiento armónico, es decir, que haya momentos de alta actividad y momentos de baja actividad. En el primer estado el 
sujeto estaría condicionado por la razón, ya que necesita ubicarse en un espacio y tiempo determinado para comprender la situación. En el segundo estado, el espectador se deja llevar por el ritmo de la historia y los artilugios propios de la narración cinematográfica. Con base en lo anterior, el analista de neuro métrica espera que la película conserve un equilibrio para que el espectador sufra un viaje de la razón a la emoción y luego a la serenidad.

De acuerdo con Frazzetto (2014), Damasio (2010), Damasio (2009), Damasio, (2001) y Damasio (1994), desde el punto de vista biológico las emociones son reacciones psicofisiológicas que representan modos de adaptación a ciertos estímulos. Dichas reacciones son mecanismos de defensa que le permiten al individuo juzgar, dado el caso, si hay peligro. Según Damasio (2010), un individuo se encuentra en estado de armonía si su organismo no ha sido alterado por una enfermedad, el ambiente u otros entes externos. Si un sujeto percibe peligro, la química de su cuerpo cambia gracias al cerebro. El cerebro secreta en la sangre cortisol u hormona del estrés y la percepción del tiempo se ralentiza porque el torrente sanguíneo se ha inundado de adrenalina. El miedo acelera el ritmo cardiaco, el pulso aumenta, las pupilas se dilatan, los poros de la piel se abren y secretan sudor. Una vez la situación llega a su fin los neurotransmisores, la serotonina y las endorfinas hacen que el cuerpo entre en estado de relajación. La secuencia descrita es lo que se espera produzca en el organismo del espectador un tráiler o una película.

\section{Discusión}

Smidts (2002) expresó que el propósito del neuromarketing era comprender mejor al cliente para llevar a cabo la comercialización de productos mediante la medición de los procesos cerebrales y, eventualmente, ayudar a un gerente a desarrollar estrategias de marketing eficaces para aumentar las ventas. Para Braidot (2010), el neuromarketing, "es una disciplina avanzada que investiga y 
estudia los procesos cerebrales que explican la conducta y la toma de decisiones de las personas” (p. 4). De acuerdo con Lindstrom (2010), el neuromarketing es "un curioso matrimonio entre el marketing y la ciencia, era la ventana que tanto había ansiado descubrir para observar el interior de la mente humana" (p. 14). De igual manera coinciden Underhill (2000), Underhill (2006), Underhill, (2007), Malfitano (2007), Pradeep (2010) y Lehrer (2011).

En consecuencia, la presente investigación concuerda en que el neuromarketing es la unión de las técnicas del marketing y las neurociencias con el objetivo de discernir o conocer la actividad cerebral de las personas para comprender aquello que incide en el proceso de decisión de compra o aceptación de un producto. Por otro lado, también se aceptan las diferentes posiciones éticas sobre los riesgos de aplicar las neurociencias en campos externos, o ajenos, a la medicina. Por ejemplo, lo que expone Monasterio (2005):

Esta explosión interdisciplinar que unifica diversas áreas en torno al conocimiento del cerebro, es cuando menos de agradecer. Es el cerebro, el sistema más complejo del universo conocido y el responsable de construir nuestra individualidad. Llegar a desvelar sus secretos es cuando menos análogo al hallazgo del Santo Grial, y por supuesto, enormemente beneficioso. Pero también se corre el riesgo de usar el conocimiento del cerebro disponible, de un modo maquiavélico. (p. 30).

Respecto al neuromarketing y la aplicación de técnicas de las neurociencias para evaluar los tráileres cinematográficos, el experimento de Hansson et al. (2008) no se llevó a cabo con el objetivo de producir películas más emocionantes o tráileres más atrayentes para el público, su interés era plenamente científico. De acuerdo con Hansson et al. (2008), "The latest advances in functional magnetic resonance imaging (fMRI ${ }^{2}$ ) offer an opportunity to measure brain activity during free viewing of films" (p. 2). Es decir, la tecnología permitía comprender

2 Los últimos avances en las imágenes de resonancia magnética funcional (fMRI) ofrecen una oportunidad para medir la actividad cerebral durante el visionado de películas (traducción nuestra). 
lo que ocurría dentro del cerebro de un espectador mientras observa una película, pero no significaba que era posible utilizar las técnicas mencionadas para producir un filme.

Sin embargo, el neuromarketing hace parte de la gran industria del cine que lidera Hollywood, por lo tanto emplea las técnicas que le ofrece la ciencia y la tecnología para lograr mayores ingresos en taquilla. De acuerdo con Hammou, Galib y Melloul (2013), "Several years later, Lacey (2010) defined neurocinema as an offshoot of neuromarketing"3 (p. 25). El neuromarketing también captó el interés de los medios de comunicación.

This offshoot of neuromarketing got intensive mass media attention, and the San Diego-based firm MindSign Neuromarketing was the first neuromarketing firm to create a revolution in this industry through the use of fMRI to test and track the impact of scenes and analyze significant activity on the prospect's brain ${ }^{4}$ (Hammou, Galib y Melloul, 2013, p. 25).

Existe una nueva perspectiva sobre la manera en que las nuevas tecnologías incidirían en la producción cinematográfica. De la misma manera, las compañías de neuromarketing se presentaban como los nuevos actores con la capacidad de subsanar las nuevas necesidades de la gran industria del cine. El éxito de la película Avatar (Cameron, 2010) demostró, según Randall (2011), que el neurocinema era un nicho rentable para Hollywood y para las empresas que se dedican a la aplicación de técnicas de neurociencia para la producción de películas. Asimismo, profesionales de diferentes áreas de la producción cinematográfica deberán pensar en el neuromarketing como un paso necesario si quieren hacer parte de la nueva tendencia.

3 Varios años más tarde, Lacey (2010) define neurocinema como una rama del neuromarketing (traducción nuestra).

4 Esta rama del neuromarketing consiguió intensiva atención en los medios de comunicación, y la empresa con sede en San Diego MindSign Neuromarketing fue la primera empresa de neuromarketing en crear una revolución en este sector a través del uso de fMRI para probar y realizar el seguimiento del impacto de las escenas y analizar una actividad significativa en la perspectiva de cerebro (traducción nuestra). 
De acuerdo con Randall (2013), CEO Innerscope realizó un experimento con más de mil personas que observaron cuarenta tráileres cinematográficos que se produjeron entre los años 2010 al 2012. El público se analizó con diversas técnicas de neurociencia que incluían el registro de la actividad cardíaca, el grado de sudoración y demás. Se encontró que existía correlación entre el contenido emocional del filme y el comportamiento de la taquilla. La experiencia explica el interés de los productores de Hollywood por el neuromarketing.

\section{Conclusiones}

El neuromarketing convoca el interés de diversidad de investigadores, de estudiantes de publicidad y negocios, al igual que en el campo de la medicina, pero muy poco hacia la investigación de los tráileres cinematográficos. Dicha área es muy específica dentro del campo de las neurociencias y es difícil encontrar información detallada y especializada, pero es posible hallar varios documentos serios que permiten aclarar términos, establecer el contexto histórico y comprender en qué consiste dicha técnica. El neuromarketing aplicado a los tráileres cinematográficos es un fenómeno de mercadeo del que aún hay mucho que decir, en particular sobre su efectividad. Las técnicas del neuromarketing tienen cierta similitud con métodos tradicionales. Por ejemplo, el Focus Group, que tiene por objetivo registrar las opiniones de un grupo de consumidores para comprender el gusto de la mayoría. En neuromarketing se emplean técnicas que registran la actividad neuronal del público para comprender el gusto y aceptación de un producto, dicho método se denomina Neuro focus.

En la cinematografía, una buena la película es el resultado de la imaginación, la sensibilidad y capacidad del director para conmover y emocionar al espectador. Hasson et al. (2008) concluyeron que la película que mayor grado de aceptación tuvo entre los participantes del experimento fue el tele film Alfred Hitchcock Presents: Bang! Your Dead (Hitchcock, Swanton \& Vosper, 1961), que dirigió Alfred Hitchcock, obra que se logró antes de que se emplearan técnicas de 
las neurociencias para comprender si la pieza era atractiva para los espectadores. Una película tiene una construcción compleja y el empleo de técnicas de las neurociencias no determina que la película sea buena o mala. Una película contiene mensajes que ayudan a que el público se sienta atraído; por ejemplo el film Avatar (Cameron, 2010) no solo tiene escenas emocionantes, además promueve la reflexión sobre la ecología, el libre desarrollo de los pueblos e incluso el derecho a la libertad, valores que unen a los hombres en torno a ideas.

En el caso de filmes de arte y ensayo o películas que utilicen estrategias narrativasmuypersonales, elespectadorbuscauna experienciaintelectual o emocional, pero no en el sentido que se ha expuesto en este artículo, sino a través del descubrimiento de preocupaciones, problemáticas y sentimientos tratados de una manera creativa. Generalmente el tipo de estética propio de dichos filmes a veces se sustenta en formas difíciles de seguir para espectadores del común. "El predominio de los filmes clásicos de Hollywood, y consecuentemente de la narración clásica, es un hecho histórico pero la historia del cine no es monolítica. Bajo diversas circunstancias, han aparecido modos diversos de narración" (Bordwell, 1996, p. 204). Lo expuesto implica que el neuromarketing no aportaría a la estética, sino que su función queda restringida al marketing y, en particular, a filmes ligados a narraciones tradicionales y géneros de acción, aventura, terror, etc. Películas en las cuales es más factible tener en cuenta las emociones del espectador.

Para terminar, es posible que el neuromarketing no incida de la misma manera en cinematografías de menor capacidad de producción y distribución. En los países menos desarrollados, la producción audiovisual es incipientey, por lotanto, no se considera que la producción cinematográfica sea un renglón importante dentro de la economía. Ya que, en dicho ámbito, la mayoría de las películas se financian con estímulos gubernamentales. El neuromarketing es una técnica que necesariamente está ligada a la gran industria de Hollywood o a países desarrollados que tienen la tecnología, el mercado y la distribución. 


\section{Referencias bibliográficas}

American Marketing Association. AMA (2013). Definition of Marketing. Recuperado de: https://www.ama.org/AboutAMA/Pages/Definition-of-Marketing.aspx

Benito, S. M., \& Guerra, V. F. (2011). Neuromarketing: tecnologías, mercado y retos. Pensar la Publicidad. Revista Internacional de Investigaciones Publicitarias, 5(2), 19-42. Recuperado de:

http://revistas.ucm.es/index.php/PEPU/article/download/37862/36630

Berrocal, J. A. J. (2013). Música y neurociencia: Un paso más en el conocimiento del ser humano. Artseduca, (4), 70-73. Recuperado de:

http://dialnet.unirioja.es/servlet/articulo?codigo $=4182408$

Berčík, J., Horská, E., Wang, W. Y. \& Chen, Y. C. (2015 March). How can food retailing benefit from neuromarketing research: a case of various parameters of store illumination and consumer response. In 143rd Joint EAAE/AAEA Seminar, March 25-27, 2015, Naples, Italy (No. 202714). European Association of Agricultural Economists. Recuperado de:

http://ageconsearch.umn.edu/bitstream/202714/2/145.pdf

Bordwell, D. (1996). La narración en el cine de ficción. Barcelona: Paidós.

Braidot, N. (2010). Neuromarketing aplicado. Science 311(5763), 935. Recuperado de: http:// files.soluciones-administrativas7.webnode.com.co/200000011-c1a05c38de/506_ Neuromarketing\%20Aplicado.pdf

Cameron, J. (Guionista/Director). (2010). Avatar. [Película]. Beverly Hills, CA, EE. UU.: 20th Century Fox Home Entertainment / Lightstorm Entertainment / Giant Studios Inc.

Damasio, A. R. (1994). El Error de Descartes: La emoción, la razón y el cerebro humano. Barcelona: Crítica.

Damasio, A. R. (2001). La sensación de lo que ocurre: Cuerpo y emoción en la construcción de la conciencia. Madrid: Editorial Debate.

Damasio, A. R. (2010). Y el cerebro creó al hombre: ¿Cómo pudo el cerebro generar emociones, sentimientos, ideas y el yo? Barcelona: Destino.

Damasio, A., \& Ros, J. (2009). En busca de Spinoza: Neurobiología de la emoción y los sentimientos. Barcelona: Crítica.

Dornaleteche, J. (2007). Definición y naturaleza del tráiler cinematográfico. Pensar la publicidad, 1(2), 99-117. Recuperado de: https://revistas.ucm.es/index.php/PEPU/article/ download/PEPUo707220099A/15662

Frazzetto, G. (2014). Cómo sentimos: Sobre lo que la neurociencia puede y no puede decirnos acerca de nuestras emociones. Barcelona: Anagrama.

García, C. A. S. (2014). Neuroética hoy. Dilemata, (15), 41-50. Recuperado de: http://dilemata.net/revista/index.php/dilemata/article/viewFile/289/310

Gardner, A. (2009). Neurocinematics: Your brain on film. Recuperado de:

http://newenglandfilm.com/magazine/2009/o6/neurocinematics-your-brain-on-film

Haines, D. (2002). Principios de neurociencia: Segunda edición. Barcelona: Elsevier Science.

Hammou, K. A., Galib, M. H., \& Melloul, J. (2013). The contributions of neuromarketing in marketing research. Journal of Management Research, 5(4), 20-33. Recuperado de: http://macrothink.org/journal/index.php/jmr/article/download/4023/3403 
Hasson, U., Landesman, O., Knappmeyer, B., Vallines, I., Rubin, N. \& Heeger, D. J. (2008). Neurocinematics: The Neuroscience of Film. Projections, 2 (1), 1-26. Recuperado de: http://www.cns.nyu.edu/ nava/MyPubs/Hasson-etal_NeuroCinematics2008.pdf

Hitchcock, A. (Director), Swanton, H. \& Vosper, M. (Guionistas). (1961). Alfred Hitchcock Presents: Bang! You’re Dead. [Película]. NY, EE.UU.: Shamley Productions / Columbia Broadcasting System (CBS)

Kernan, L. (2004). Coming attractions: Reading American movie trailers. Austin: University of Texas Press.

Kotler, P. \& Armstrong, G. (2013). Fundamentos de marketing. 11 ed. México D.F.: Pearson Educación.

Lacey, L. (2010). Movies that make you love them. Recuperado de: http://www.theglobeandmail.com/arts/film/movies-that-make-you-love-them/article4313328/

Lehrer, J. (2011). Cómo decidimos: Y cómo tomar mejores decisiones. Barcelona: Paidós.

Lienhart, R., Pfeiffer, S. \& Effelsberg, W. (1997). Video abstracting. Communications of the ACM, 40(12), 54-62. Recuperado de: http://videoanalysis.org/Prof._Dr._Rainer_ Lienhart/Source_Code_files/cacm.pdf

Lindstrom, M. (2010). Compradicción: Verdades y mentiras de por qué compramos. Barcelona: Ediciones Gestión 2000.

Madan, C. (2010). Neuromarketing: the next step in market research? Eureka, 1 (1), 34-42. Recuperado de: https://ejournals.library.ualberta.ca/index.php/eureka/article/download/7786/6493.

Maestú, F., Quesney, F., Ortiz, T., Fernández, A., Campo, P. \& Amo, C. (2003). Cognición y redes neurales: una nueva perspectiva desde la neuroimagen funcional. Rev Neurol, 37(10), 962-966. Recuperado de: http://www.neurologia.com/pdf/Web/3710/ p100962.pdf

Malfitano, C. O. (2007). Neuromarketing: Celebrando negocios y servicios. Buenos Aires: Ediciones Granica.

Medical News Today. MNT. (2013). What is neurosciencie? Recuperado de: http://www. medicalnewstoday.com/articles/248680.php

Monasterio, A. (2005). Las implicaciones morales de la neuroeconomía. Frónesis. 12 (2), 25 - 36. Recuperado de: http://www.produccioncientifica.luz.edu.ve/index.php/fronesis/ article/viewFile/2976/2975

Morin, C. (2011). Neuromarketing: The New Science of Consumer Behavior. Society, 48 (Issue 2), 131-135. Recuperado de: http://fac.ksu.edu.sa/sites/default/files/neuromarketing_1.pdf

Pons, E. G. (2012). La manipulación temporal en los tráileres de cine: articulación de un nuevo tiempo narrativo. Estudios sobre el Mensaje Periodístico, 18, 433-441. Recuperado de: http://revistas.ucm.es/index.php/ESMP/article/download/40998/39248

Pradeep, A. K. (2010). The buying brain: Secrets for selling to the subconscious mind. Hoboken, N.J: Wiley.

Palacios, L. (2002). Breve historia de la electroencefalografía. Acta Neurológica Colombiana, 2, 104-7. Recuperado de: http://www.acnweb.org/acta/2002_18_2_104. pdf

Ramachandran, V. S. \& Hirstein, W. (1999). The science of art: A neurological theory of aesthetic experience. Journal of consciousness Studies, 6(6-7), 15-51. Recuperado de: http://www.imprint.co.uk/rama/art.pdf. 
Randall, K. (2011). Rise of neurocinema: How Hollywood studios harness your brainwaves to win oscars. Recuperado de: http://www.fastcompany.com/1731055/rise-neurocinema-how-hollywood-studios-harness-your-brainwaves-win-oscars

Randall, K. (2013). How your brain can predict blockbusters. Recuperado de: http://www. fastcompany.com/3006186/how-your-brain-can-predict-blockbusters

Smidts, A. (2002). Kijken in het brein: Over de mogelijkheden van neuromarketing. Recuperado de: http://repub.eur.nl/pub/308/

Soriano, M. C. \& Guillazo, B. G. (2007). Fundamentos de neurociencia. Barcelona: Editorial UOC.

Society of neuroscience. (2015). About Neuroscience. Recuperado de: http://www.sfn.org/ about/aboutneuroscience?pagename=whatIsNeurosciene.

Spurlock, M. (Director), Spurlock, M \& Chilnick, J. (Guionistas). (2011). The greatest movie ever sold. [Película]. Los Ángeles, CA: Snoot Entertainment / Warrior Poets.

Tirado, D. M. (2013). Fundamentos de marketing. Recuperado de: http://repositori.uji.es/ xmlui/bitstream/handle/10234/49394/s74.pdf?sequence $=3$.

Underhill, P. (2000). Por qué compramos: La ciencia del shopping. Barcelona: Ediciones Gestión 2000.

Underhill, P. (2006). Shopping mania. Milano: Sperling \& Kupfer.

Underhill, P. (2007). El placer de comprar: 24 horas en un centro comercial. Barcelona: Ediciones Gestión 2000.

Vecchiato, G. (2013). Neuroelectrical brain imaging tools for the study of the efficacy of TV advertising stimuli and their application to neuromarketing. Berlin: Springer.

Verbinski, G. (Director) \& Logan, J. (Guion). (2011). Rango. [Película]. Hollywood, CA: Paramount Pictures / Nickelodeon Movies / Blind Wink / GK Films.

Wiseman, L. (Director) \& Bomback, M. (Guion). (2007). Die Hard 4: Live Free or Die Hard. [Película]. Los Ángeles, CA, EE.UU.: 2oth Century Fox.

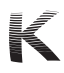

